\title{
Assessment of Methods of Decrease in Efficiency per Copy Recognition of Means \\ of a Radio Communication
}

\author{
Juriy L. Koziratsky, Alexey V. Ivantsov*, \\ Vladimir V. Ivantsov and Ervand A. Mamadganyan \\ Military Education and Research Centre of Military-Air Forces \\ "Military-Air Academy \\ Named After Professor N.E. Zhukovsky and Yu.A. Gagarin» \\ 54a Starykh Bolshevikov Str., Voronezh, 394064, Russia
}

Received 15.03.2016, received in revised form 01.02.2017, accepted 10.02.2018

The reasons of emergence of the individual technical unmasking signs of radio-electronic means are considered. Estimates of dependence of informational content of the individual technical unmasking sign on a total error of measurement at various fixed values of a mean square deviation are carried out. It is shown that for reduction of informational content of a sign possibly application of unification of parameters of transmitters and deliberate change of parameters of a signal.

Keywords: per copy recognition, the technical unmasking sign, informational content.

Citation: Koziratsky Ju.L., Ivantsov A.V., Ivantsov V.V., Mamadganyan E.A. Assessment of methods of decrease in efficiency per copy recognition of means of a radio communication, J. Sib. Fed. Univ. Eng. technol., 2019, 12(1), 88-96. DOI: 10.17516/1999494X-0030.

(C) Siberian Federal University. All rights reserved

This work is licensed under a Creative Commons Attribution-NonCommercial 4.0 International License (CC BY-NC 4.0).

* Corresponding author E-mail address: kagan13@yandex.ru 


\title{
Оценка методов снижения эффективности
}

\section{поэкземплярного распознавания средств радиосвязи}

\author{
Ю.Л. Козирацкий, А.В. Иванцов, \\ В.В. Иванцов, Е.А. Мамаджанян \\ Военный учебно-научный изентр Военно-воздушных сил \\ «Военно-воздушная академия \\ имени профессора Н.Е. Жуковского и Ю.А. Гагарина» \\ Россия, 394064, Воронеж, ул. Старых Большевиков, 54а
}

Рассмотрень причины возникновения индивидуальных технических демаскирующчих признаков радиоэлектронных средств. Проведены оценки зависимости информативности индивидуального технического демаскирующчего признака от суммарной погрешности измерения при различных фиксированных значениях среднеквадратичного отклонения. Показано, что для уменьшения информативности признака возможно применение унификации параметров передатчиков и умышленное изменение параметров сигнала.

Ключевые слова: поэкземплярное распознавание, технический демаскирующчий признак, информативность.

Функционирование средств радиосвязи в составе современных систем управления характеризуется большим количеством и разнообразием типов мобильных связных радиоэлектронных средств (РЭС), функционирующих как автономно, так и в составе сложных объектов. Современные связные РЭС способны излучать различные виды сигналов в широком частном диапазоне. Кроме того, излучения гражданских РЭС имеют большую степень подобия с излучениями РЭС военного назначения [1].

При отсутствии достоверной априорной информации это приводит к снижению вероятности распознавания типов РЭС, ошибкам в определении их принадлежности и, как следствие, к неверной количественной и качественной оценке радиоэлектронной обстановки (РЭО). Исключить указанные ошибки или существенно уменьшить их влияние на оценку РЭО позволяет поэкземплярное распознавание РЭС, под которым понимается распознавание сигналов отдельного экземпляра РЭС среди совокупности однотипных средств независимо от изменения режимов их работы и местоположения [2].

Очевидно, что наиболее сложна задача выделения экземпляров именно среди однотипных средств, имеющих одинаковые структуру, элементную базу, конструкцию, диапазоны изменения параметров сигналов и принципы применения. Поэкземплярное распознавание РЭС основано на выявлении и измерении присущих каждому отдельному экземпляру РЭС индивидуальных технических демаскирующих признаков (ТДП). Эти признаки обусловлены наличием индивидуальных для каждого экземпляра РЭС конструктивных, технологических и эксплуатационных факторов, влияющих на процессы формирования и излучения радиосигналов.

Цель стать - разработка методического подхода для оценки информативности индивидуальных ТДП РЭС и методов снижения эффективности поэкземплярного распознавания.

Для поэкземплярного распознавания РЭС могут быть использованы как основные, так и неосновные излучения передатчиков. К неосновным излучениям относятся побочные (излуче- 
ния на гармониках и субгармониках, комбинационные и паразитные излучения) и внеполосные излучения [3].

Кроме технических параметров сигналов, индивидуальную информацию о РЭС содержат так называемые структурные характеристики, среди которых можно выделить две группы:

параметры внутриимпульсной модуляции;

характеристики «тонкой» структуры сигнала.

Так, при распознавании РЭС, излучающих ЧМ-сигналы, в качестве экземплярных признаков могут быть выбраны величина девиации частоты, величина базы сигнала, форма огибающей радиоимпульса, а также вид и параметры закона изменения частоты внутри импульса. Основными признаками поэкземплярного распознавания для фазоманипулированных сигналов могут являться величина длительности элементарной посылки, величина скачка фазы и параметры закона модуляции (вид кодовой последовательности, основание и значность кода, структура кодообразующего полинома, начальная фаза кода и т.п.). Кроме того, для средств радиосвязи в качестве индивидуальных признаков могут служить структурные признаки: наличие синхрогруппы и служебных комбинаций, наличие и состав адресной части сообщения, наличие и вид закрытия сообщения, вид передаваемой информации.

К характеристикам «тонкой» структуры импульсных радиосигналов обычно относят параметры формы переходных процессов установления амплитуды, частоты и фазы высокочастотных колебаний на участках фронта и среза импульсов, а также флюктуации амплитуды, частоты и фазы высокочастотных колебаний на участке вершины импульса. Важное место наряду с рассмотренными выше признаками, непосредственно связанными с характеристиками излучений передатчиков РЭС, при поэкземплярном распознавании занимают косвенные признаки, не связанные с параметрами сигналов, но определяемые конструктивными и технологическими особенностями каждого экземпляра РЭС. Некоторые косвенные признаки могут быть получены путем измерения параметров сигналов.

К ним, например, относятся характеристики диаграммы направленности антенны (ширина основного лепестка диаграммы направленности антенны, наличие, количество, уровень, ширина боковых лепестков диаграммы направленности).

Таким образом, даже сравнительно небольшой обзор индивидуальных особенностей излучений передатчиков РЭС и косвенных признаков показывает, что многие из рассмотренных выше характеристик сигналов могут быть положены в основу создания системы поэкземплярного распознавания РЭС [4]. Но окончательный отбор признаков осуществляется путем оценивания их информативности с обязательным учетом устойчивости значений признаков в процессе функционирования РЭС.

Под информативностью признака понимают характеристику его разделяющей способности. Устойчивость (стабильность) признака - его способность сохранять неизменным свое среднее значение при различных изменениях режима работы РЭС, перерывах в работе, а также при передислокации РЭС.

Требование устойчивости признаков распознавания обусловлено тем, что практически все современные типы РЭС имеют возможность изменения режимов работы по несущей частоте, длительности и периоду повторения импульсов. Использование подобных параметров сигналов в качестве признаков поэкземплярного распознавания неизбежно приведет к потере инфор-

$$
-90-
$$


мации об экземплярах РЭС, особенно если эти средства подвижные или транспортируемые. Для таких РЭС смена значений параметров сигнала, являющихся признаками распознавания, и передислокация в перерыве между наблюдениями приводят к полной потере информации об экземплярах.

Оценка информативности признаков может осуществляться на основе критерия Байеса или с использованием теории информации. Однако в этом случае необходимо полное знание априорной информации о параметрах признаков, что в реальных условиях затруднительно. Поэтому для оценки информативности при поэкземплярном распознавании необходимо использовать более наглядный показатель, чувствительный к стабильности параметров передающих устройств, точности измерений и состоянию среды распространения.

В качестве такого показателя выступает отношение

$$
J=\frac{\Delta S_{0}}{\Delta S_{p r}}
$$

где $\Delta S_{0}$ - среднеквадратичное отклонение значения ТДП РЭС данного типа; $\Delta S_{p r}$ - среднеквадратичное отклонение погрешности измерения.

Теоретически показатель информативности может изменяться в очень широких пределах, характеризуя идеальную информативность признака. При высокой степени идентичности параметров передатчиков $\Delta S_{0} \rightarrow 0$, следовательно, информативность $J \rightarrow 0$. При высокой точности измерения $\Delta S_{p} \rightarrow 0$ и некоторой постоянной $\Delta S_{0}$ информативность $J \rightarrow \infty$. В реальных условиях фактическая информативность всегда ниже вследствие влияния тракта распространения, помех и устойчивости признака во времени.

Дисперсия суммарной погрешности средства измерения определяется выражением

$$
\Delta S_{u}^{2}=\Delta S_{p r}^{2}+\Delta S_{T}^{2}+\Delta S_{P}^{2}+\Delta S_{N}^{2}
$$

где $\Delta S_{p r}^{2}$ - дисперсия погрешности измерения признака; $\Delta S_{T}^{2}-$ дисперсия погрешности измерения, вносимого трактом распространения радиоволн; $\Delta S_{P}^{2}$ - дисперсия погрешности измерения, обусловленной помехами; $\Delta S_{N}^{2}$ - дисперсия погрешности измерения, обусловленной нестабильностью признака во времени [5].

Соответственно, показатель реальной информативности признака будет иметь вид

$$
J_{r}=\frac{\Delta S_{0}}{\sqrt{\Delta S_{p r}^{2}+\Delta S_{T}^{2}+\Delta S_{P}^{2}+\Delta S_{N}^{2}}}=K \cdot J,
$$

где $K$ - коэффициент потери информативности, определяемый как

$$
K=\frac{1}{\sqrt{1+\left(\frac{\Delta S_{T}}{\Delta S_{p r}}\right)^{2}+\left(\frac{\Delta S_{P}}{\Delta S_{p r}}\right)^{2}+\left(\frac{\Delta S_{N}}{\Delta S_{p r}}\right)^{2}}} .
$$

Коэффициент потери информативности указывает на степень влияния мешающих факторов на качество признаков. Для успешного распознавания существует определенное значение отношения $\left(\Delta S_{0} / \Delta S_{p r}\right)$. Оно зависит от длительности излучения, надежности распознавания, отношения сигнал/шум и может быть представлено в виде функционала 


$$
\left(\frac{\Delta S_{0}}{\Delta S_{p r}}\right)_{n}=\varphi\left(N, t_{\text {изл }}, P_{d}, q\right),
$$

где $N$ - число РЭС, подлежащих распознаванию; $t_{\text {изл }}-$ длительность излучения; $P_{d}-$ надежность распознавания; $q$ - соотношение сигнал/шум.

Успешность распознавания по некоторому признаку обеспечивается при выполнении соотношения

$$
\left(\frac{\Delta S_{0}}{\Delta S_{p r}}\right)_{n} \leq \frac{\Delta S_{0}}{\Delta S_{u}}
$$

Отсюда $\Delta S_{p r}^{2}+\Delta S_{T}^{2}+\Delta S_{P}^{2}+\Delta S_{N}^{2} \leq \frac{\Delta S_{0}^{2}}{\left(\Delta S_{0} / \Delta S_{p r}\right)_{n}^{2}}$.

Преобразовав это неравенство, получим

$$
\frac{J}{J_{n}} \geq \sqrt{1+\frac{\Delta S_{\Sigma}^{2}}{\Delta S_{p r}^{2}}}
$$

где $\Delta S_{\Sigma}^{2}=\Delta S_{T}^{2}+\Delta S_{P}^{2}+\Delta S_{N}^{2}-$ суммарная погрешность измерений, обусловленная мешающими факторами.

Признак может считаться информативным, если его информативность больше или равна некоторой необходимой информативности

$$
J \geq J_{n}
$$

Учитывая, что неравенства (4) и (6) эквивалентны, можно сказать, что признак является информативным, если оценка среднеквадратичной погрешности измерения удовлетворяет условию

$$
\frac{\Delta S_{0}}{J_{n}} \geq \Delta S_{u}
$$

которое можно представить как

$$
\left(\frac{\Delta S_{0}}{J_{n}}\right)^{2} \geq \Delta S_{u}^{2}
$$

На рис. 1 изображены графики зависимости информативности индивидуального ТДП РЭС от суммарной погрешности измерения при различных фиксированных значениях среднеквадратичного отклонения ТДП.

Анализ зависимостей позволяет сделать вывод о том, что при равной суммарной погрешности измерения информативность признака определяется величиной среднеквадратичного отклонения ТДП, то есть степенью унификации РЭС. При этом с ростом $\Delta S_{u}$ значение информативности менее чувствительно к изменению $\Delta S_{0}$.

Предположим, что все систематические погрешности измерений определены и результат скорректирован посредством введения поправок. Поскольку точность определения поправок не является абсолютной, вследствие их введения возникают дополнительные случайные погрешности, приводящие к увеличению общей дисперсии. 


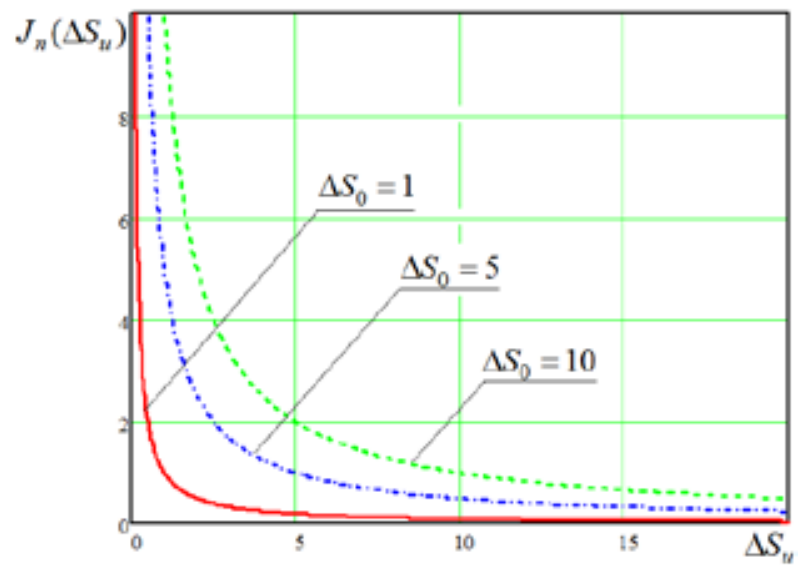

Рис. 1. Графики зависимости информативности индивидуального технического демаскирующего признака РЭС от суммарной погрешности измерения

Fig. 1. Schedules of dependence of information content of individual technical demasking feature of the electronic means of the total measurement error

\section{Представим дисперсию погрешности в виде суммы}

$$
\Delta S_{u}^{2}=\Delta S_{\bar{x}}^{2}+\Delta S_{q}^{2},
$$

где $\Delta S_{\bar{x}}^{2}-$ дисперсия среднего арифметического результата измерения; $\Delta S_{q}^{2}-$ дисперсия поправки систематической погрешности измерения.

Величины $\Delta S_{\bar{x}}^{2}$ и $\Delta S_{q}^{2}$ определяются выражениями

$$
\left\{\begin{array}{l}
\Delta S_{\bar{x}}^{2}=\sum_{i=1}^{m_{1}} \Delta S_{\bar{x}_{i}}^{2} ; \\
\Delta S_{q}^{2}=\sum_{i=1}^{m_{2}} \Delta S_{q_{i}}^{2},
\end{array}\right.
$$

где $\Delta S_{\bar{x}_{i}}^{2}$ - дисперсия среднего арифметического, обусловленная $i$-й случайной погрешностью; $\Delta S_{q_{i}}^{2}$ - дисперсия поправки $i$-й систематической погрешности; $m_{1}-$ количество случайных погрешностей; $m_{2}$ - количество систематических погрешностей.

Дисперсия среднего арифметического под действием $i$-й случайной погрешности определяется выражением

$$
\Delta S_{\bar{x}_{i}}^{2}=\frac{1}{n_{i}\left(n_{i}-1\right)} \sum_{j=1}^{i}\left(x_{j}-\bar{x}\right)^{2},
$$

где $n_{i}=\left[t_{\text {изм }} / \tau_{k_{i}}\right]-$ число некоррелированных измерений при $i$-й случайной погрешности; $t_{\text {изм }}$ время измерения; $\tau_{k_{i}}$ интервал корреляции $i$-й случайной погрешности; [...] - целая часть числа.

\section{Соответственно,}

$$
\Delta S_{\bar{x}_{i}}^{2}=\frac{\tau_{k_{i}}}{t_{\text {изм }}\left(n_{i}-1\right)} \sum_{j=1}^{n_{i}}\left(x_{j}-\bar{x}\right)^{2} .
$$


Преобразуя (8) с учетом (9), (10) и (12), получим выражение условия информативности признака:

$$
\left(\frac{\Delta S_{0}^{2}}{J_{n}^{2}}-\sum_{i=1}^{m_{2}} \Delta S_{q_{i}}^{2}\right) \cdot t_{\text {изм }} \geq \sum_{i=1}^{m_{1}} \frac{\tau_{k_{i}}}{\left(n_{i}-1\right)} \cdot \sum_{j=1}^{n_{i}}\left(x_{j}-\bar{x}\right)^{2} .
$$

Выражение (13) связывает дисперсию средних арифметических значений параметров передатчиков с необходимой информативностью признака, длительностью излучения и оценкой дисперсии единичного измерения.

Анализ неравенства (13) позволяет сделать вывод, что при $J_{n}=$ const признак является более информативным при большей дисперсии $\Delta S_{0}^{2}$, большем времени измерения $t_{\text {изм }}$ и меньших дисперсиях систематических погрешностей и единичных измерений. Следовательно, для уменьшения информативности признака возможно применение следующих способов:

унификации параметров передатчиков;

умышленного изменения параметров сигнала.

При унификации передатчиков необходимо добиваться идентичности параметров сигналов на выходе РЭС, т.е. найти технические решения для уменьшения разброса математических ожиданий значений параметров передатчиков, т.е. для уменьшения $\Delta S_{0}^{2}$ (левая часть неравенства (13)). Уменьшение $\Delta S_{0}^{2}$ в первую очередь определяется точностью установки номинальных значений унифицируемых параметров и их стабильности во времени, что зависит от уровня развития технологической базы. Следует отметить, что необходимая идентичность должна быть не только достигнута при производстве РЭС, но и поддерживаться в ходе эксплуатации. Однако вследствие объективных причин достичь абсолютной идентичности нельзя. Выражение (13) позволяет произвести оценку степени целесообразной унификации параметров сигналов, показывающую, что если некоторый унифицированный параметр имеет дисперсию $\Delta S_{0}^{2}$ и не ограничивает необходимое время измерения существенно больше, чем другие параметры, то степень его унификации может считаться удовлетворительной и информативным его считать нельзя.

Снижение информативности путем умышленного изменения параметров сигнала может существенно затруднить поэкземплярное распознавание, если параметр сигнала изменяется непрерывно таким образом, что мгновенные значения признаков для разных РЭС пересекаются, изменения одинаковы для всех РЭС данного типа и закон их распределения исключает появление вторичных признаков. При использовании этого способа определяющее значение имеют выбор закона распределения параметра, установление возможных пределов изменения параметра и скорости его изменения.

Очевидно, что для снижения информативности признаков необходимо изменять параметры по случайному закону, обладающему наибольшей неопределенностью. Из всех известных случайных законов максимальную энтропию имеет равновероятный закон распределения, однако он же наиболее труден в реализации, поэтому из соображений простоты реализации может быть выбран другой закон, например усеченный нормальный. При этом не следует ожидать за время включения РЭС четко выраженной периодичности изменения параметра и больших отклонений параметра от номинальных. Ограничение по отклонению от номинала определяется помехоустойчивостью приема, а исключение периодичности обусловлено возможностью 
появления вторичных признаков, которые могут быть установлены путем корреляционного анализа.

Изменение значения параметра во времени и незнание его фактического отклонения от математического ожидания в момент измерения эквивалентно увеличению погрешности измерения. То есть умышленное изменение параметра добавляет дополнительное слагаемое к правой части неравенства (13), что приводит к снижению информативности признака, которая уменьшается с увеличением интервала корреляции и ростом разброса его единичных измерений. Ограничение предела изменения параметра сверху определяется допустимой степенью уменьшения помехоустойчивости. Нижний предел можно вычислить из (13). Положим, что погрешности измерения, не связанные с их умышленным изменением, отсутствуют. Тогда выражение (13) примет вид

$$
\frac{\Delta S_{0}^{2}}{J_{n}^{2}} \cdot t_{\text {изм }} \geq \frac{\tau_{k_{i}}}{(n-1)} \cdot \sum_{i=1}^{n}\left(x_{i}-\bar{x}\right)^{2},
$$

или $\frac{\Delta S_{0}^{2}}{J_{n}^{2}} \geq \frac{1}{n} \cdot \Delta S_{x^{\prime}}^{2}$, где $\Delta S_{x^{\prime}}^{2}-$ дисперсия погрешности измерения, вызванная умышленным изменением параметра.

Для того чтобы при умышленном изменении параметра выполнялось условие информативности признака, необходимо

$$
\Delta S_{x^{\prime}}^{2} \leq \frac{\Delta S_{0}^{2}}{J_{n}^{2}} n .
$$

Для равновероятного закона максимальное отклонение параметра от его математического ожидания определим по формуле

$$
\Delta x_{\max }^{\prime}=\sqrt{3} \Delta S_{x^{\prime}} \leq \sqrt{3 n} \frac{\Delta S_{0}}{J_{n}} .
$$

Для нормального усеченного закона

$$
\Delta x_{\max }^{\prime} \approx(2-3) \Delta S_{x^{\prime}}
$$

Из (17) и (18) следует, что признак не будет достаточно информативным, если

$$
\left.\Delta x_{\max }^{\prime}\right\rangle(2-3) \frac{\Delta S_{0}}{J_{n}}
$$

Информативность признака обеспечит успешное распознавание, если

$$
\Delta x_{\max }^{\prime} \leq \sqrt{3 n} \frac{\Delta S_{0}}{J_{n}} .
$$

Выбор скорости изменения параметра определяется тем, что уменьшение скорости ведет к увеличению интервала корреляции и, соответственно, к уменьшению числа независимых измерений $n$, что, в свою очередь, приводит к росту погрешности измерений и к снижению информативности признака. С другой стороны, чрезмерно медленное изменение 
значений параметров излучений может дать возможность прогнозирования значений параметров.

Таким образом, рассмотрение возможных методов снижения информативности признаков позволяет сделать выводы о том, что наиболее эффективно снижение информативности путем уменьшения среднеквадратичного отклонения значения ТДП РЭС данного типа $\Delta S_{0}^{2}$, т.е. унификацией передатчиков РЭС. В ходе умышленного изменения параметров РЭС к уменьшению $\Delta S_{0}^{2}$ добавляется увеличение погрешности измерения $\Delta S_{x^{\prime}}^{2}$.

\section{Список литературы}

[1] Козирацкий Ю.Л., Будников С.А., Иванцов А.В. и др. Модели информационного конфликта средств поиска и обнаружения. М.: Радиотехника, 2013. 232 с. [Kozirazki Yu. L., Budnikov S. A., Ivantsov A.V., etc. Model of information conflict of means of search and discovery. Moscow, Radiotexnika, 2013. 232 p. (in Russian)]

[2] Куприянов А.И., Петренко П.Б., Сычев М.П. Теоретические основы радиоэлектронной разведки. М: Изд-во МГТУ им. Н.Э. Баумана, 2010. 381 с. [Kupriyanov A. I., Petrenko P. B., Sychev M. P. Theoretical foundations of electronic intelligence. M., Izd-vo MGTU im. N.E. Bauman, 2010. 381 p. (in Russian)]

[3] Гавриленко И.И. Радиопередаюшие устройства. М.: Транспорт, 1983. 368 с. [Gavrilenko I. I. Radio Transmitting devices. Moscow, Transport, 1983. 368 p. (in Russian)]

[4] Козирацкий Ю.Л., Донцов А.А., Иванцов А.В. и др. Модели пространственного и частотного поиска. М.: Радиотехника, 2014. 344 с. [Kozirazki Y. L., Dontsov A. A., Ivantsov A.V., etc. Model spatial and frequency search. Monograph; ed. by L. Kozirazki. Moscow, Radiotexnika, 2014. 344 p. (in Russian)]

[5] Дворяшин Б.В. Основы метрологии и радиоизмерения. М.: Радио и связь, 1993. 320 с. [Dvoryashin B. V. Fundamentals of metrology and radio measurement. M., Radio and communication, 1993. 320 p. (in Russian)] 